$\begin{array}{ll} & \text { Etnográfica } \\ \text { etnográfica } & \text { Revista do Centro em Rede de Investigação em }\end{array}$

Antropologia

vol. $16(2) \mid 2012$

Vol. $16(2)$

\title{
Ta'zie (le théâtre religieux) vs Noruz (la nouvelle année et ses rituels): les enjeux de la politique du patrimoine immatériel de l'humanité en Iran
}

Ta'zie (o teatro religioso) vs Noruz (o Ano Novo e os seus rituais): implicações da política do património imaterial da humanidade no Irão

\section{Christian Bromberger}

\section{OpenEdition}

Journals

Édition électronique

URL : https://journals.openedition.org/etnografica/1561

DOI : 10.4000/etnografica. 1561

ISSN : 2182-2891

\section{Éditeur}

Centro em Rede de Investigação em Antropologia

Édition imprimée

Date de publication : 1 juin 2012

Pagination : 407-417

ISSN : 0873-6561

Référence électronique

Christian Bromberger, «Ta'zie (le théâtre religieux) vs Noruz (la nouvelle année et ses rituels): les enjeux de la politique du patrimoine immatériel de l'humanité en Iran », Etnográfica [En ligne], vol. 16 (2) | 2012, mis en ligne le 26 juin 2012, consulté le 12 février 2022. URL : http:// journals.openedition.org/etnografica/1561; DOI : https://doi.org/10.4000/etnografica.1561

\section{(c) (†) 8}

Etnográfica is licensed under a Creative Commons Attribution-NonCommercial 4.0 International License. 


\section{Ta'zie (le théâtre religieux) vs Noruz (la nouvelle année et ses rituels): les enjeux de la politique du patrimoine immatériel de l'humanité en Iran}

\section{Christian Bromberger}

En 2005, l'Iran a soumis à l'UNESCO deux dossiers de candidature de chefs d'œuvre du patrimoine immatériel, l'un sur le ta'zie ou shabikhâni (une forme de tragédie religieuse propre à l'islam chiite), le second sur les rituels de Noruz (la nouvelle année, qui coïncide avec l'équinoxe de printemps). L'Iran retira finalement la candidature du ta'zie et ne maintint que celle de Noruz qui fut rejetée par le jury de l'UNESCO au motif que le dossier était incomplet, ce qui suscita amertume et polémique. Trois questions se posent: Pourquoi la candidature du ta'zie fut-elle retirée in extremis? Pourquoi une telle insistance et une telle agitation autour de Noruz? Pourquoi accorde-t-on une telle importance en Iran à l'inscription d'un bien culturel sur la liste des chefs d'œuvre du patrimoine immatériel?

MOTS-CLÉS: patrimoine immatériel, Iran, ta'zie, Noruz.

Ta'zie (o teatro religioso) vs Noruz (o Ano Novo e os seus rituais): implicações da política do património imaterial da humanidade no Irão - Em 2005, o Irão apresentou à UNESCO dois dossiês de candidatura de obras-primas do património imaterial, um sobre o ta'zie ou shabikhâni (uma forma de tragédia religiosa específica do Islão xiita) e o segundo sobre os rituais de Noruz (o Ano Novo, que coincide com o equinócio da primavera). O Irão acabou por retirar a candidatura do ta'zie e manteve apenas a do Noruz, que viria a ser rejeitada pelo júri da UNESCO com o argumento de que o dossiê estaria incompleto, o que causou animosidade e polémica. Três questões se colocam: por que razão a candidatura do ta'zie foi retirada in extremis? Porque houve tanta insistência e agitação em torno do Noruz? Porque se atribui no Irão tamanha importância à inscrição de um bem cultural na lista das obras-primas do património imaterial?

PALAVRAS-CHAVE: património imaterial, Irão, ta'zie, Noruz.

BROMBERGER, Christian (bromberger.christian@gmail.com) - Université de Provence, France 


\section{AU-DELÀ DU SOUCI LÉGITIME DE PRÉSERVER “TRÉSORS” ET “CHEFS} d'œuvre" immatériels, quels sont les enjeux extra-patrimoniaux qui se faufilent sous les demandes de classement par l'UNESCO ? En examinant de récents projets présentés par l'Iran, je voudrais me livrer à une analyse ethnographique du sens de ces candidatures, l'ethnographie étant, selon les mots de Clifford Geertz, "an enterprise [...] whose aim is to render obscure matters intelligible by providing them with an informing context" (1983: 152).

En 2005, l'Iran a soumis à l'UNESCO deux dossiers de candidature de chefs d'œuvre du patrimoine immatériel, l'un sur le ta'zie ou shabikhâni (une forme de tragédie religieuse propre à l'islam chiite), le second sur les rituels de Noruz (la nouvelle année, qui coïncide avec l'équinoxe de printemps). Présentons brièvement ces deux candidatures avant d'en scruter les coulisses.

\section{TA'ZIE}

Le premier dossier, préparé par le Centre des arts dramatiques de Téhéran (Center of Dramatic Arts, Tehran), incluait une série de documents sur les représentations théâtrales de l' "histoire-mythe" de Kerbala et des évènements qui y sont associés. En octobre 680, le jour de âshurâ (le 10 moharram, premier mois de l'année musulmane), Hoseyn, fils de 'Ali et petit fils du prophète Mohammad, fut massacré, avec l'ensemble de ses partisans et des membres masculins de sa famille (à l'exception de deux d'entre eux), par les troupes du calife omeyyade, Yazid, sur le site de Kerbala, à proximité de l'Euphrate, dans l'actuel Irak. La douloureuse passion et le martyre de Hoseyn, troisième imam des chiites, constituent l'horizon majeur, le paradigme de la religiosité populaire et la source vive de la division entre chiites et sunnites. ${ }^{1}$ La commémoration du supplice du "prince des martyrs" (seyyed al-shohâda) s'exprime à travers des rituels dolorisants pendant les dix premiers jours de moharram et atteignent leur paroxysme le 10 de ce mois: processions de pénitents se flagellant avec les paumes des mains, des chaînes ou encore se meurtrissant naguère le cuir chevelu avec une épée ou un sabre, ${ }^{2}$ prônes, cantiques et représentations théâtrales commémorant le drame de Kerbala scandent ces jours de deuil et d'affliction. Le jour même de âshurâ, au terme du cycle cérémoniel, les scènes finales du drame sont rejouées par les fidèles dans la cour de nombreux sanctuaires: l'incendie des tentes des membres de la sainte famille, le meurtre de l'imam dont le cheval blanc est couvert de sang, les berceaux abandonnés des enfants martyrisés...

l Le premier imam est 'Ali, le second Hasan, frère de Hoseyn. Les chiites iraniens reconnaissent douze imams, qui ont tous péri dans des conditions tragiques, à l'exception du douzième qui a disparu en 874 ; son "occultation" dure encore et les fidèles en attendent la parousie.

2 Cette pratique est interdite par les autorités religieuses depuis 1994. 
Le ta'zie est donc une sorte de rituel dramatisé, un genre théâtral semblable aux mystères chrétiens du Moyen-Âge, qui représentaient la Passion du Christ. Il existe plusieurs centaines de variantes de ta'zie, la plupart anonymes, écrits dans une langue simple, joués le plus souvent par des acteurs non professionnels, accompagnés par un petit orchestre. ${ }^{3}$ Les représentations ont lieu sur des places publiques ou dans des locaux (tekkye) spécialement consacrés aux préparatifs des cérémonies et à ce genre de manifestations. Quel qu'en soit le thème, et a fortiori si le ta'zie évoque directement le drame de Kerbala, les représentations opposent deux classes de personnages: les bons et les vertueux (Hoseyn et les siens), emblèmes de la justice et de la pureté, vêtus de vert, psalmodiant gravement leurs plaintes et louant le sacrifice rédempteur de l'imam; les méchants (Yazid et ses soldats, Shemr, l'auteur du monstrueux forfait, le meurtre de Hoseyn), symbolisant la tyrannie et l'oppression, habillés de rouge ou de mauve et disant leur texte sur un ton saccadé, les yeux menaçants et écarquillés. Les acteurs (ta'ziekhân) sont tous masculins, même s'ils incarnent des personnages féminins. Ils ne s'identifient pas, à proprement parler, à leur rôle ; ils en sont de simples imitateurs qui manifestent occasionnellement, quand ils jouent des "méchants", le dégoût que leur inspire le rôle qu'ils tiennent.

Le $t a^{\prime} z i e$ est fortement ancré dans les traditions nationales et religieuses iraniennes. Il a été codifié sous la grande dynastie safavide (1501-1722), qui érigea le chiisme en religion d'état, s'est développé au XVIII ${ }^{m e}$ siècle sous les Afshar et les Zand et a atteint son apogée au XIX ${ }^{\text {ème }}$ siècle sous les Qajar. De splendides tekkye furent alors édifiés, dont le célèbre tekkye dowlat ("tekkye du gouvernement") à Téhéran, qui fut détruit sous la dynastie pahlavi (19251979). Si les Qajar avaient encouragé et patronné les représentations de ta'zie pour asseoir culturellement leur pouvoir, les souverains pahlavi (Reza et son fils Mohammed Reza), créateurs d'un État moderne tourné vers l'Occident et valorisant le grandiose passé préislamique de leur empire, interdirent ce genre théâtral, qui ne fut réhabilité qu'à la fin de la dynastie, quand le dernier shah et son épouse engagèrent une politique de valorisation des arts et traditions populaires iraniens à la faveur de somptueux festivals internationaux. Ce mouvement de réhabilitation fut encouragé par des intellectuels nationalistes qui soulignaient volontiers, à tort ou à raison, les origines préislamiques du personnage de Hoseyn, héros héritier, selon eux, de Siyavosh, victime innocente de la haine des hommes dont le Livre des Rois de Ferdowsi (X⿳亠丷⿵冂丶 ${ }^{\text {se }}$ siècle) relate la légende et le supplice.

3 Citons, parmi les plus célèbres, les ta'zie de Moslem (envoyé par Hoseyn et tué ainsi que ses enfants par Ibn Ziyad, le commandant des troupes de Yazid), de Horr (soldat valeureux qui se repent et se rallie à la cause de l'imam), de la mort de Zeynab (la courageuse sœur de l'imam), de Mokhtar qui fit périr, cinq ans après le drame, les responsables du massacre de Kerbala, celui des Quatre oiseaux et de la jeune fille juive, où une jeune aveugle recouvre la vue, une goutte de sang de l'imam Hoseyn, transportée par des oiseaux lui étant miraculeusement tombée sur les yeux... 
Dans un contexte général d'iconophobie et de théâtrophobie (beaucoup moins marqués cependant qu'en islam sunnite), le clergé chiite eut des positions variables au fil du temps, tantôt condamnant, tantôt acceptant ce genre de représentations où sont mis en scène de saints personnages et où s'intercalent des épisodes merveilleux et parfois comiques. Malgré leurs réticences sur l'orthodoxie de ce spectacle, les autorités de la République islamique l'autorisent, considérant que ces représentations offrent aux fidèles une occasion de renforcer et de manifester leur foi. Devant les drames auxquels ils assistent, les spectateurs ne demeurent, en effet, pas passifs: ils prennent à partie et insultent les "méchants", se lamentent en se frappant la poitrine lors des épisodes les plus tragiques... Le ta'zie est, en définitive, un symbole majeur du monde chiite, attesté non seulement en Iran, mais aussi en Irak, dans les émirats, au Liban, en Inde, etc., si bien que l'on pourrait qualifier de ta'ziestân l'ensemble des régions qui partagent ces références communes. ${ }^{4}$ Cette commune appartenance à un même courant religieux peut entraîner une solidarité politique et humanitaire, comme c'est le cas entre le gouvernement iranien et les Hazaras (chiites) d'Afghanistan, comme c'est aussi le cas avec le Hezbollah libanais ou encore avec les chiites du sud de l'Irak. On a ainsi pu parler, sans doute abusivement, d'un "arc chiite" au Moyen-Orient.

Le ta'zie correspond parfaitement aux six critères retenus par l'UNESCO pour définir un chef d'œuvre du patrimoine immatériel. Il s'agit d'un genre original, enraciné dans une tradition culturelle, symbole d'identité, qui associe textes littéraires, musique instrumentale et scénographie de qualité; bien qu'en déclin, le ta'zie demeure une tradition vivante, prisé différemment d'une catégorie de spectateurs à l'autre (hommes ou femmes, jeunes ou vieux, lettrés et gens du peuple..., les uns et les autres étant plus ou moins sensibles à tel ou tel aspect du drame: la tyrannie et l'injustice des "méchants", les relations mère-enfant, la qualité littéraire du texte, le jeu des acteurs...); mais c’est aussi un genre menacé de disparition ou d'artificialisation esthétisante, concurrencé par les spectacles modernes (séries télévisées, etc.), dont les savoirs se perdent, et qui ne fait l'objet d'aucune mesure de protection. Voilà donc un candidat idéal à la consécration par l'UNESCO.

4 Ta'zie est un mot emprunté à l'arabe qui signifie à l'origine "déploration, témoignage de condoléances". Il est ici transcrit phonétiquement conformément aux règles adoptées par la plupart des iranologues (voir Lazard 1991). Le sens du mot, comme la pratique, à laquelle il se réfère, varient selon les régions et les nations où les chiites sont implantés. En Iran et au Liban, le mot désigne le théâtre religieux; au Liban encore, mais avec une autre acception, les processions des pénitents; en Irak, les rituels de moharram; en Inde, les répliques en miniature du mausolée de Hoseyn que portent les fidèles lors des processions. Mais, quelles que soient ces variantes, le mot se réfère toujours au drame de Kerbala et à la passion de Hoseyn. Il existe sur le ta'zie de nombreux travaux comprenant une abondante bibliographie (voir, récemment, Shahriari 2006 et Chelkowski 2009). 


\section{NORUZ}

Sur le chemin de sa reconnaissance comme chef d'œuvre du patrimoine immatériel et comme symbole majeur de l'identité iranienne, le ta'zie a cependant un redoutable concurrent, Noruz ("le jour nouveau”), qui inaugure le début de l'année à l'équinoxe de printemps. Ce calendrier solaire tranche avec le calendrier lunaire musulman et est un des symboles forts de la spécificité du monde iranien, remontant à l'Antiquité. Selon la tradition, Noruz perpétuerait et commémorerait le jour de la création du monde par Ahura Mazda. De nombreux rites et rituels, similaires à ceux qui inaugurent le printemps dans plusieurs sociétés de l'Ancien monde, scandent et célèbrent la nouvelle année: le grand nettoyage de la maison (khâne tekâni), l'achat de vêtements neufs, la décoration d'œufs, la mise en germination de graines de blé, d'orge ou de lentilles qu'on laisse pousser dans une assiette et qui donnent le sabze (verdure), la préparation d'une pâtisserie (samanu) à base de suc de blé et de sucre, ces deux préparations faisant partie des haft sin (les sept "s"), c'est-à-dire des sept denrées dont le nom commence par un "s" que l'on place sur une nappe, étalée par terre, pendant ce temps inaugural. La veille du dernier mercredi de l'année (dit le "mercredi rouge", chahâr shanbe suri), on allume un feu que les membres de la maisonnée ou du quartier sautent en disant: "Zardi o ranjuri-ye man be to, sorxi o xorâmi-ye to be man" ("Ma pâleur et mon chagrin pour toi, ta rougeur et ta gaieté pour moi"). D’autres pratiques ponctuent cette période de transition: mascarades, chants (noruzkhâni), séances de divination, vols rituels, assortis de vœux, effectués par les jeunes garçons, etc. Ce cycle de la nouvelle année s'achève le 13 du premier mois, le sizdah bedâr ("le 13 dehors!"). Pour conjurer le malheur associé au chiffre 13, les familles quittent alors leur maison et vont pique-niquer dans un lieu verdoyant. Signe de la clôture du temps de Noruz, on jette dans la mer ou dans un cours d'eau le sabze et l'on défait la nappe des haft sin.

Tandis que, on l'a dit, le ta'zie est volontiers présenté par les intellectuels nationalistes comme un genre plongeant ses origines dans la mythologie préislamique, Noruz, coutume attestée dans l'Antiquité, a été islamisée au fil des siècles: ce jour inaugural est censé coïncider avec celui de l'apparition de l'archange Gabriel au prophète Mohammad, avec celui de l'investiture par le même prophète Mohammad de 'Ali comme son successeur légitime ou encore avec celui de la future parousie de l'imam caché (voir note 1). Quelles que soient ces nuances, ta'zie et Noruz ont des profils nettement tranchés que l'on pourrait décrire en termes d'oppositions structurales: au genre essentiellement religieux qu'est le $t a$ 'zie, évoquant la souffrance et suscitant la peine, s'oppose Noruz, un fête fondamentalement laïque et symbole d'allégresse. Les deux rites peuvent, certaines années, se chevaucher, le premier s'inscrivant dans un calendrier lunaire, le second dans un calendrier solaire. Dans ce cas, 
les manifestations de liesse sont réduites au minimum et s'effacent derrière la commémoration du deuil. En 2006, par exemple, le quarantième jour suivant âshurâ, qui fait aussi l'objet d'un rituel d'affliction, coïncidait avec Noruz. Des affiches officielles proposaient un compromis au bénéfice du prince des martyrs: "Noruz-e man bar Hoseyn ast" ("Mon Noruz est pour Hoseyn"). Ce compromis témoignait cependant d'un assouplissement par rapport à la première décennie ayant suivi la Révolution de 1979; pendant ces dix premières années, les autorités islamiques, soucieuses de propager une idéologie chiite révolutionnaire, avaient combattu et tenté de réduire au minimum les coutumes "spécifiquement iraniennes" qui avaient été valorisées par la dynastie pahlavi au nom du nationalisme culturel. A partir des années 1990, qui inaugurèrent une libéralisation relative du régime islamique, la fierté nationale reprit ses droits et le folklore "spécifiquement iranien" fut même partiellement réhabilité pour lutter contre "l'invasion culturelle occidentale" ("tahâjom-e farhangi-ye qarb"). Ethnologues et, plus généralement, intellectuels nationalistes s'engouffrèrent dans cette brèche si bien que les séminaires, les conférences, les livres sur Noruz foisonnèrent. Une sorte de "Noruzmania" semblait s'être emparée des milieux culturels. Elle eut de forts échos, j'y reviendrai, dans les pays d'Asie centrale et du Caucase, englobés dans l'aire historique d'extension de la civilisation iranienne et partageant le même calendrier. C'est ce que l'on appelle le sarzamin-e Irân (le monde iranien) et ce que l'on pourrait tout aussi bien appeler, en raison de cette référence commune, le Noruzestân.

Tout comme le ta'zie, Noruz présente toutes les qualités d'un bon candidat à l'inscription au patrimoine immatériel de l'humanité: ancrage traditionnel, originalité, symbole d'identité, intérêt historique et esthétique... tous les qualificatifs requis par l'UNESCO peuvent être appliqués sans peine à ce rite de renouveau qui, contrairement au ta'zie, n'est pas menacé de disparition.

\section{LES ENJEUX D'UNE CANDIDATURE}

L'Iran avait donc présenté, en 2005, les candidatures de ces deux indiscutables "chefs d'œuvre", l'une, celle du ta'zie, à un titre strictement national, la seconde, celle de Noruz, en association avec neuf autres pays partageant plus ou moins cette coutume: l'Afghanistan, l'Ouzbekistan, la Kirghizie, le Tajikistan, le Kazakhstan, le Pakistan, l'Inde (où la communauté zoroastrienne est d'origine iranienne), l'Azerbaïjan, la Turquie. L'Iran retira finalement la candidature du ta'zie et ne maintint que celle de Noruz qui fut rejetée par le jury de l'UNESCO au motif que le dossier était incomplet, ce qui suscita amertume et polémique.

Au terme de cette présentation factuelle, trois questions se posent? Pourquoi la candidature du ta'zie fut-elle retirée in extremis? Pourquoi une telle insistance et une telle agitation autour de Noruz? Pourquoi accorde-t-on une 
telle importance en Iran à l'inscription d'un bien culturel sur la liste des chefs d'œuvre du patrimoine immatériel, cet intérêt étant moindre dans d'autres pays $?^{5} \mathrm{Au}$ fond, ces candidatures et les démarches qui les accompagnent ne nous en apprennent-ils pas autant sur les débats politico-culturels qui agitent un pays que sur ce que sont des "chefs d'œuvre" du patrimoine immatériel?

C'est dans le contexte des années 2000, sous la présidence de Mohammad Khatami, un réformateur, que furent préparées ces candidatures. Les responsables de la culture et du patrimoine sont alors animés d'un souci d'ouverture qui se combine avec une fierté nationale qui valorise les spécificités iraniennes et minimise les solidarités islamiques, fussent-elles chiites (l'anti-arabisme est une composante majeure de ce nationalisme). De façon significative, le dossier du ta'zie, qui aurait pu comporter des comparaisons avec d'autres pays chiites où le genre est attesté (au Liban, par exemple), était exclusivement centré sur l'Iran. On lui préféra celui sur Noruz, rappelant, dans un contexte de lutte d'influence avec la Russie et la Chine en Asie centrale, l'importance historique de la civilisation et de l'empire iraniens. Il est vrai que, de la Mésopotamie à l'ouest de la Chine, l'empreinte du "grand Iran" (Iran-e bozorg) est forte. Outre l'Iran, l'Afghanistan et le Tajikistan ont le persan pour langue nationale; une importante minorité de la population d'Ouzbekistan le parle. La plupart des États du Caucase étaient inclus dans l'empire d'Iran jusqu'au premier quart du XIX ${ }^{m e}$ siècle. L'insistance sur cette histoire partagée et sur ce patrimoine commun s'est traduite par une multitude d'initiatives culturelles. En 2004 s'est tenu à Téhéran, sous l'égide de la commission iranienne de l'UNESCO, un atelier sur le rôle des femmes dans la transmission et la sauvegarde du patrimoine immatériel: il réunissait des participants des mêmes pays "parents" (à l'exception de l'Inde), auxquels s'étaient joints des représentants de l'Arménie et de la Géorgie. En Iran, plusieurs livres et conférences ont été récemment consacrés à Noruz, le premier congrès portant explicitement sur ce thème s'étant déroulé en mars 2000 à Persépolis, la capitale affective des empereurs achéménides. À plusieurs de ces manifestations participent les "pays frères", comme à Sari, dans le nord de l'Iran en avril 2006, où se déroula un imposant "Festival du patrimoine commun aux peuples riverains de la mer Caspienne et d'Asie centrale", dont Noruz fut le symbole glorifié. Lors de ces différents colloques, les responsables iraniens du patrimoine présentent leur pays comme "la maison du père" (khâne-ye pedari), tout en multipliant les précautions rhétoriques pour ne pas choquer leurs voisins. Ils rappellent ainsi, à l'occasion, que les centres de la civilisation iranienne n'ont pas toujours été en Iran. Boukhara, Samarcande

5 Pour prendre un exemple extrême de ce désintérêt, je citerai le cas de Tarascon, une petite ville du sud de la France, dont le maire n'était même pas au courant de la candidature de la fête locale de la Tarasque et de son inscription, conjointement avec d'autres cérémonies belges célébrant des Géants, sur la prestigieuse liste des "chefs d'œuvre". 
ne sont-ils pas situés dans l'actuel Ouzbekistan? Cette carte du Noruzsestân se superpose, de façon significative, à celle de l'Economic Cooperation Organization (ECO), fondée en 1985 par l'Iran, le Pakistan et la Turquie, basée à Téhéran, et élargie, depuis 1992, à l'Afghanistan, aux nouveaux États d'Asie centrale et à l'Azerbaïjan. Ces États revendiquent d'autant plus cette référence commune à Noruz que la célébration de la fête était interdite du temps de l'Union soviétique et a symbolisé la fin du communisme et l'indépendance nationale. De 1926 à 1988, les rites de Noruz n'étaient, en effet, accomplis que clandestinement, dans un cadre familial. Une des premières mesures prises par les nouveaux États dès leur indépendance, voire dès la perestroïka, fut la restauration de Noruz, rapidement déclarée fête nationale. Tel fut le cas en Ouzbekistan, où un décret présidentiel de février 1989 consacra cette réhabilitation; suivirent la création d'une "Fondation Navruz", puis celle d'une "Navruz International Charity Foundation" en 1992. En Afghanistan, la fête fut bannie par les Soviétiques, puis par les Talibans, avant d'être de nouveau célébrée avec ferveur après le renversement de leur régime.

Dans ce chorus à la gloire de Noruz, l'autre grande puissance régionale, la Turquie, qui a de forts liens culturels et linguistiques avec plusieurs nouvelles Républiques du Caucase et d'Asie centrale, semble jouer sa propre partition. Ses représentants participent peu aux conférences et aux projets communs célébrant Noruz. Au moment où se discutait ce dossier, l'intégration dans l'Union européenne était manifestement plus à l'ordre du jour à Ankara et à Istanbul que le panturquisme. Par ailleurs, jouer les seconds rôles dans une opération culturelle placée sous l'égide de l'Iran n'était et n'est sans doute pas non plus du goût des dirigeants turcs. Est-ce à dire pour autant que le gouvernement turc se désintéresse de Noruz? Non pas, mais pour bien d'autres raisons que la constitution d'un Noruzestân à dominante iranienne.

Au début du XX⿳亠丷⿵冂丶 ${ }^{2}$ siècle, la célébration de Newroz était tombée en désuétude chez les Kurdes de Turquie (qui sont, rappelons-le, une population d'origine iranienne), quand des intellectuels nationalistes l'érigèrent en fête nationale à la fin des années 1910.6 Dans les années 1960, les militants de la cause kurde s'emparèrent de cette date et de ce symbole pour organiser manifestations et mobilisation. C'est, par exemple, le jour de Newroz qu'en 198434 militants s'immolèrent par le feu dans la prison militaire de Diyarbakir. Dans cette course aux symboles mobilisateurs, la minorité religieuse alévie, fortement implantée dans l'est de la Turquie, ne demeura pas en reste. Sans doute, une partie d'entre elle avait coutume de célébrer Nevruz, mais la revitalisation de la fête a coïncidé avec la poussée revendicative et politique du mouvement aléviste dans les années $1990 .^{7}$ Comme dans les interprétations des chiites

6 Sur les avatars de Nevruz en Turquie, voir l'excellente synthèse de Massicard (2002: 410-414).

7 Sur les relances et revitalisations de traditions, voir Bromberger et Chevallier (2004). 
iraniens, la date est désormais censée correspondre à l'anniversaire ou à l'investiture de 'Ali par Mahomet. Ces réappropriations (à chacun son Noruz!) n’ont pas laissé sans réaction les dirigeants turcs. Quand une coutume ou un rite devient un symbole oppositionnel, deux solutions se présentent pour le pouvoir en place: ou les interdire, ce qui risque de susciter amertume et rébellion, ou en revendiquer le patronage, voire la paternité. Comme les souverains qajar avaient organisé de somptueux ta'zie pour asseoir leur popularité et brider l'opposition religieuse, les dirigeants turcs célèbrent officiellement Noruz depuis le milieu des années 1990, cherchant ainsi à couper l'herbe sous les pieds des Kurdes et des Alévis. Ils affirment sans ambages qu'il s'agit là d'une tradition originelle turque, ce que viennent confirmer complaisamment des ethnologues et des historiens. La preuve de cette turcité de la coutume n'est-elle pas fournie par son attestation au Kazakhstan et en Ouzbekistan, chez les "Turcs de l'extérieur" (où, en fait, Noruz est un apport de la civilisation iranienne)?

Noruz, ses rites, ses coutumes culinaires, les chansons, les récits, les croyances qui l'entourent méritent sans nul doute d'être distingués et médaillés par l'UNESCO. Il s'agit d'un ensemble de traditions originales qu'il convient de préserver. Mais il serait naïf de penser que seuls des arguments culturels sont à l'origine de cette candidature qui serait uniquement le fruit d'un consensus d'experts. Volonté hégémonique, revendications nationalistes et sécessionnistes, contre-offensives étatiques tiennent une large part dans la dynamique et les ratés du projet. Il y eut d'excellents élèves (l'Iran, le Kazakhstan, l'Ouzbekistan, par exemple), d'autres semblèrent moins zélés, tel la Turquie, sans doute peu pressée de voir Kurdes et Alévis se prévaloir d'un symbole (revendicatif) reconnu par l'UNESCO. Tout dans ces ratés ne s'explique, bien sûr, pas par des arrière-pensées politiques. Mais le choix ou la ratification par un ou plusieurs États d'un candidat au chef d'œuvre invite tout autant à s'interroger sur la qualité d'un bien culturel et du dossier qui le présente que sur le sens et les intentions sous-jacents au projet. Vu l'effervescence culturelle du début des années 2000 en Iran, rendue possible par l'accession au pouvoir du réformateur Mohammad Khatami, vu le renouveau, dans ce contexte, des initiatives pour valoriser le passé préislamique du pays, vu le souci d'affirmer le leadership de la civilisation spécifiquement iranienne dans cette partie du monde, il n'est pas étonnant que le projet Noruz ait été préféré au projet ta'zie.

L'intérêt particulier, voire l'acharnement, de certains États à faire bénéficier leur patrimoine du prestigieux label de l'UNESCO méritent aussi réflexion. Sans doute une telle reconnaissance ouvre-t-elle de remarquables possibilités de développement du tourisme, au risque même d'entraîner une "folklorisation" de la pratique que l'on entend protéger. Il est, par exemple, significatif qu'en Iran patrimoine et tourisme soient regroupés dans la même organisation. Mais, au-delà, c'est la volonté de reconnaissance d'un État sur la scène internationale, tout autant que celle d'un bien culturel, qui rend compte de l'atmosphère 
passionnelle qui entoure ces candidatures. Ce souci de distinction est d'autant plus fort que le régime du pays qui présente le dossier souffre d'une mauvaise réputation. Pour les opposants, souvent réfugiés dans les ONG, une telle reconnaissance est le moyen de proclamer que leur pays a un autre visage que celui qu'exhibent leurs gouvernants. Pour le pouvoir en place, c'est une occasion inespérée de faire parler positivement du pays qu'il dirige, de redorer son blason, de se donner un supplément d'âme, voire de faire diversion. Les réactions qui suivirent, en Iran, le rejet de l'inscription de Noruz sur la liste des chefs d'œuvre du patrimoine immatériel témoignent de l'intensité de ces enjeux symboliques. On condamna l'opacité des procédures de l'UNESCO; certains virent dans ce refus le fruit d'un complot israélien; d'autres mirent en cause l'arriération de "pays frères" qui n'avaient pas tenu leurs engagements, etc. ${ }^{8}$

Au total, la reconnaissance d'un bien culturel comme "patrimoine immatériel de l'humanité" soulève des enjeux qui débordent de loin le champ de la seule expertise ethnographique. Ou plutôt elle offre à l'ethnographe une occasion privilégiée d'exercer son art dans toute sa plénitude: non pas seulement en évaluant l'exactitude d'informations factuelles, mais en s'interrogeant sur l'arrière-plan conjoncturel de choix et de décisions. Les processus qui amènent à sélectionner un "candidat" plutôt qu'un autre, les relations entre les organismes qui établissent et présentent les dossiers (centres de recherche, ONG, commission(s) nationale(s) de l'UNESCO, État(s), etc.) constituent un domaine particulièrement fécond pour l'investigation ethnographique. L'UNESCO, en adoptant en 2003 la Convention pour la sauvegarde du patrimoine immatériel, a ouvert de remarquables possibilités pour le sauvetage de biens culturels menacés, mais aussi un nouveau champ de recherche et de controverse anthropologiques. Ainsi la distinction, discutable, entre patrimoines "immatériel" et "matériel" sollicite la réflexion et la critique ethnographiques. Noruz avec sa nappe décorée, avec ses préparations culinaires, relève-t-il du patrimoine "matériel" ou "immatériel"? Même question au sujet de la gastronomie française qui a été récemment honorée par l'UNESCO et dont l'amateur de bonne chère pourrait s'inquiéter qu'elle ne soit qu' “immatérielle”. À l'inverse, la notion même de "culture matérielle", adoptée par de nombreux anthropologues, apparaît très discutable. Ne vaudrait-il pas mieux parler d' "expression matérielle de la culture" et nuancer ainsi l'opposition entre "matériel" et "immatériel" qui apparaît souvent artificielle?9 On a justement défini les

8 La candidature de Noruz au patrimoine immatériel de l'humanité sera, en définitive, acceptée en septembre-octobre 2009, lors de l'Intergovernmental Committee for the Safeguarding of the Intangible Heritage qui s'est tenu à Abu Dhabi. Le projet retenu fut présenté par l'Iran, l'Azerbaijan, la Kirghizie, l'Ouzbekistan, le Pakistan, le Tajikistan et la Turquie. Quant au ta'zie, à la suite d'une nouvelle candidature, il sera inscrit au patrimoine immatériel de l'humanité, lors de la session qui s'est tenue à Nairobi (Kenya) en novembre 2010.

9 Je m'explique sur cette notion dans un dialogue avec M.-L. Gélard (Bromberger et Gélard 2012). 
objets comme de la pensée solidifiée et Mary Douglas et Baron Isherwood (1978) disent tout aussi justement que les objets rendent visibles les catégories de la culture. Dissocier ainsi le matériel et le symbolique en deux champs autonomes me semble mal venu. Un calice relève-t-il du matériel ou du symbolique? Voilà une des nombreuses questions que pose l'extraordinaire programme mis en œuvre par l'UNESCO.

\section{BIBLIOGRAPHIE}

BROMBERGER, Christian, et D. CHEVALLIER, 2004, “De la métamorphose de la châtaigne à la renaissance du Carnaval: relances de traditions dans l'Europe contemporaine", in C. Bromberger, D. Chevallier et D. Dossetto (orgs.), De la métamorphose de la châtaigne à la renaissance du Carnaval: Relances de traditions dans l'Europe contemporaine. Die, À Die, 11-18.

BROMBERGER, Christian, et Marie-Luce GÉLARD, 2012, "Culture matérielle ou expression matérielle de la culture?”, Ethnologie Française, 42 (2), 350-357.

CHELKOWSKI, P., 2009, “Ta'zia”, Encyclopaedia Iranica, online.

DOUGLAS, M., et Baron ISHERWOOD, 1978, The World of Goods: Towards an Anthropology of Consumption, Harmondsworth, Penguin.

GEERTZ, Clifford, 1983, "The way we think now: toward an ethnography of modern thought", in Clifford Geertz, Local Knowledge: Further Essays in Interpretive Anthropology. New York, Basic Books.

LAZARD, G., 1991, Dictionnaire Persan-Français. Leiden, Brill.

MASSICARD, Élise, 2002, Construction identitaire, mobilisation et territorialité politique: Le mouvement aléviste en Turquie et en Allemagne depuis la fin des années 1980. Paris, Institut d'études politiques, thèse de doctorat.

SHAHRIARI, K., 2006, Breaking Down Borders and Bridging Barriers: Iranian Taziyeh Theatre, University of New South Wales, thèse de doctorat. 